\title{
COMPUTERIZED SPOILED TOMATO DETECTION
}

\author{
Dhanabal T $\mathbf{T}^{1}$, Debabrata Samanta ${ }^{2}$ \\ ${ }^{1,2}$ Assistant Professor, Dept. of MCA, Acharya Institute of Technology, Bangalore
}

\begin{abstract}
In this paper illustrates the improvement of a low cost machine vision system using webcams and image processing algorithms for defect detection and sorting of tomatoes The sorting decision was based on three features extracted by the different image processing algorithms. This methodology based on the color features, which used for detecting the BER from good tomatoes. Two methods were developed for decision based sorting. The color image threshold method with shape factor was found efficient for differentiating good and defective tomatoes. The overall accuracy of defect detection attained was 94 and 96.5\% respectively. Comparison of the results is also presented in this paper.
\end{abstract}

Keywords: Dither Image, Stem Image, Histogram, Tomato.

\section{INTRODUCTION}

Agriculture is described as the art and science of growing tomatoes, fruits, vegetables, and trees and shrubs effecting in the increase of the minds and sentiments of individuals and the enrichment and health of community's civilization. As for explore on tomato harvesting robots, some works have been going on. To accomplish the automation by the robot, however, many troubles must be solved because the agricultural crop, which it handles, is slight and is not unvarying. Since the agricultural environment and crop have diversity, the robot needs dexterity and flexibility. It also contains narrated services in plant conservation, landscape restoration, landscape and garden design. This range of food, medicinal, environmental, and social merchandises and services are all fundamental to enlarging and continuing human health and well-being. Agriculturists concern the knowledge, skills, and technologies exploited to breed intensively produced plants for human food and non-food uses and for personal or social needs.

The aim of this paper is to new approach for detecting damaged tomatoes. From histogram we extract the difference between the intensity among the original tomatoes and the diseases affected tomatoes.

\section{CORE OF APPROACH}

Computerization of eminence control is vastly significant as saving time and expenses is constantly a necessity in industrial applications. According to specialists, the following problems arise in quality control by individuals:

- Low exactitude due to features such as ambient enlightenment intensity, person's fatigue, etc

- Lack of homogeneity caused by aforementioned factors

- Lack of truthfulness as results of former problems
Application of synthetic vision not only helps diminution of identification time in classification process but also contributes to arrangement of classification, leading to further homogeneity in quality assessments in Tomatoes Industries. Digital Image Processing has produced a centre of consideration in the information industry and in society as a whole in recent years, due to wide availability of huge amount of image information and the impending need for turning such data into useful information and acquaintance. The information and awareness achieved can be used for relevance ranging from market analysis, fraud detection, to production control and science exploration.

Images of different tomatoes are captured using a digital camera in the similar lighting conditions and with identical the background. The intensity of the input image is estimated, which is a key appraise for the defect detection. Segmentation of defects is proposed at pixel level in spatial domain, therefore for each pixel of the tomatoes, its intensity value is used as a local feature. By the applications of assorted edge detecting, the contour is sketched over an input Tomato image.

\section{METHODOLOGY}

\subsection{Image Enhancement}

The aim of image enhancement is to recuperate the interpretability or responsiveness of information in images for human viewers, or to provide 'better' input for other computerized image processing techniques. For this purpose different Tomato images are captured from several Agricultural Research farms, Hessaraghatta Lake, Bangalore.

\subsection{Image Pre-processing}

Pre-processing methodologies use a small neighborhood of a pixel in an input image to get a brightness value in the output 
image. Such pre-processing operations are also called filtration.

Local pre-processing methodologies can be divided into the two different groups according to the goal of the processing: Smoothing suppresses noise or other small fluctuations in the image; equivalent to the suppression of high frequencies in the frequency domain. Unfortunately, smoothing also blurs all sharp edges that bear important information about the image. Gradient operators are rooted in local derivatives of the image function. Derivatives are bigger at locations of the image where the image function undergoes rapid changes. The aim of gradient operators is to indicate such locations in the image. Gradient operators suppress low frequencies in the frequency domain.

\subsection{Image segmentation}

The Sobel operator achieves a 2-D spatial gradient measurement on an image and so emphasizes regions of high spatial frequency that correspond to edges. Typically it is used to discover the approximate absolute gradient magnitude at apiece point in an input grayscale Tomato image.

\subsection{Histogram Draw}

Usually, in image processing resolution of an image is the total number of pixels in the image. The original resized image is converted to gray image such that the pixels corresponding to the leaf image are same. Then we plot the histogram for calculating the change in the pick value.

\subsection{Stem Draw}

A two-dimensional stem plot displays data as lines extending from a baseline along the $\mathrm{x}$-axis. A circle (the default) or other marker whose y-position represents the data value terminates each stem.

stem(Y) plots the data sequence $\mathrm{Y}$ as stems that extend from equally spaced and automatically generated values along the $\mathrm{x}$-axis. When $\mathrm{Y}$ is a matrix, stem plots all elements in a row against the same $\mathrm{x}$ value.

stem $(\mathrm{X}, \mathrm{Y})$ plots $\mathrm{X}$ versus the columns of $\mathrm{Y}$. X and $\mathrm{Y}$ must be vectors or matrices of the same size. Additionally, $X$ can be a row or a column vector and $\mathrm{Y}$ a matrix with length $(\mathrm{X})$ rows.

\subsection{Dither Image}

Convert image, increasing apparent color resolution by dithering.

$\mathrm{X}=$ dither(RGB, map) creates an indexed image approximation of the RGB image in the array RGB by dithering the colors in the colormap map. The colormap cannot have more than 65,536 colors.
$\mathrm{X}=$ dither(RGB, map, Qm, Qe) creates an indexed image from RGB, where Qm specifies the number of quantization bits to use along each color axis for the inverse color map, and Qe specifies the number of quantization bits to use for the color space error calculations. If $\mathrm{Qe}<\mathrm{Qm}$, dithering cannot be performed, and an undithered indexed image is returned in $\mathrm{X}$.

\subsection{Bar}

A bar graph displays the values in a vector or matrix as horizontal or vertical bars. Syntax is given below,

Syntax

$$
\operatorname{bar}(\mathrm{Y})
$$

$\operatorname{bar}(\mathrm{Y})$ draws one bar for each element in $\mathrm{Y}$. If $\mathrm{Y}$ is a matrix, bar groups the bars produced by the elements in each row. The $\mathrm{X}$-axis scale ranges from 1 up to length $(\mathrm{Y})$ when $\mathrm{Y}$ is a vector, and 1 to $\operatorname{size}(Y, 1)$, which is the number of rows, when $\mathrm{Y}$ is a matrix. The default is to scale the $\mathrm{x}$-axis to the highest $\mathrm{x}$-tick on the plot, (a multiple of 10, 100, etc.).

\section{FLOW OF WORK}

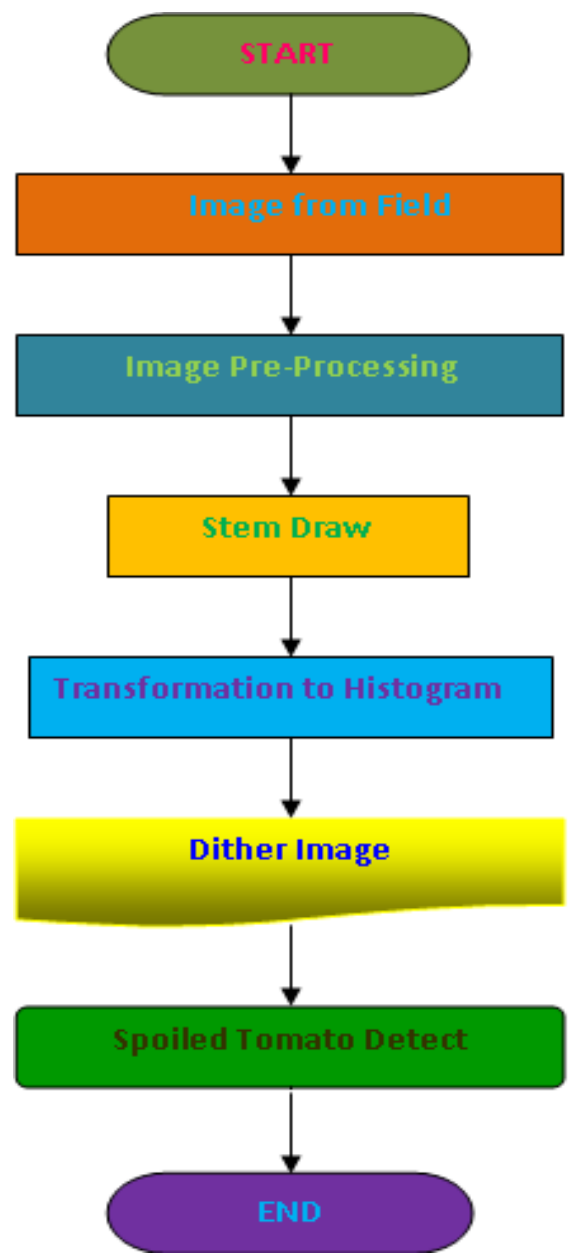




\section{RESULTS AND DISCUSSION}

The methodology has been assessment with tomato images acquired from a real greenhouse considered as an uncontrolled environment. The images are taken the undergrowth of tomato plants and some of them contained more than a single leaf. We tried to take the undergrowth in its natural state without attempting to evade shadows or overlapping leaves. To test the algorithm, 40 images were randomly selected. All of the 40 images were tested by algorithm and their results were compared and 4 result of tomato images are given below.
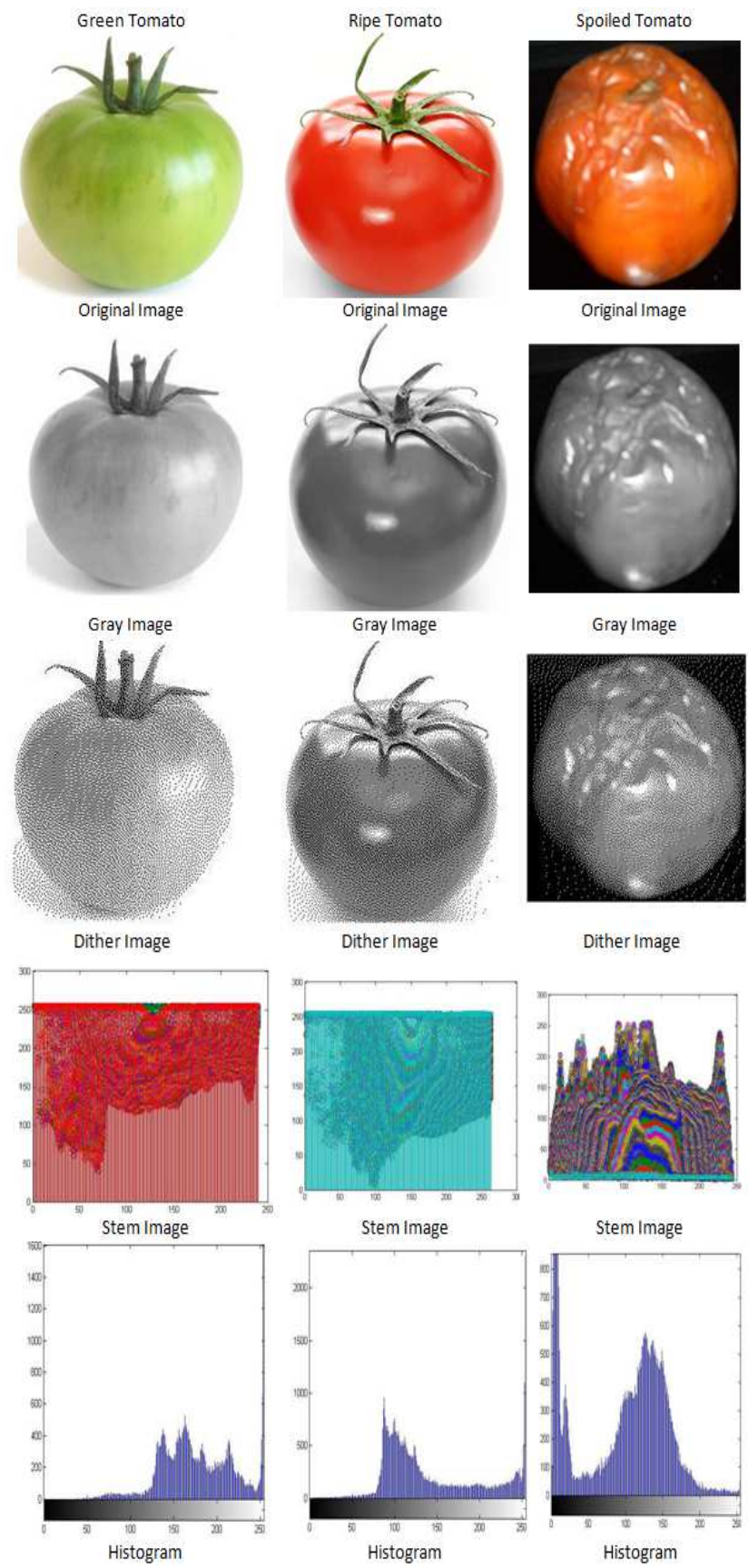

Original Image

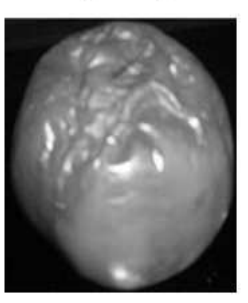

Gray Image
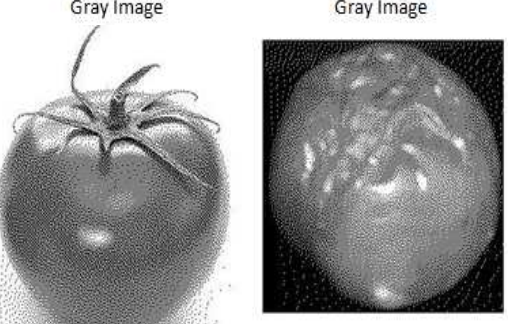

Dither Image

Dither Image
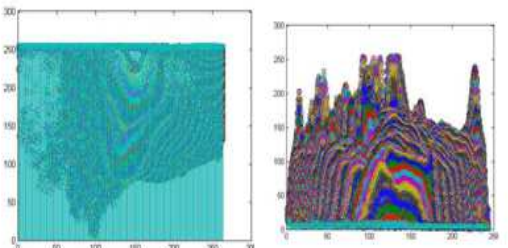

Stem Image

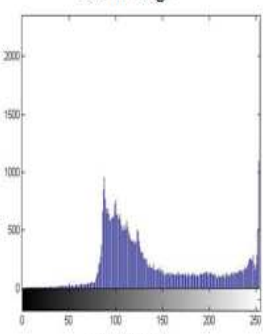

Histogram
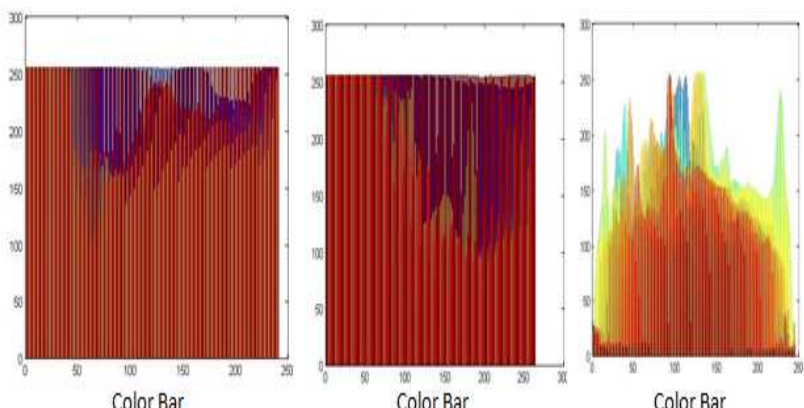

Color Bar
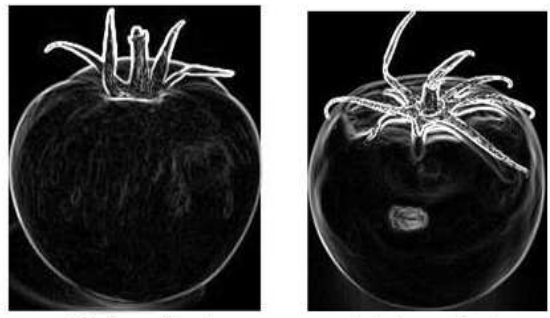

Sobel gradient

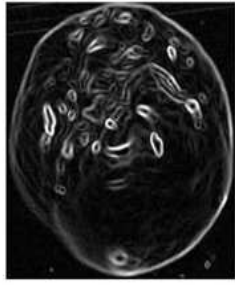

Sobel gradient
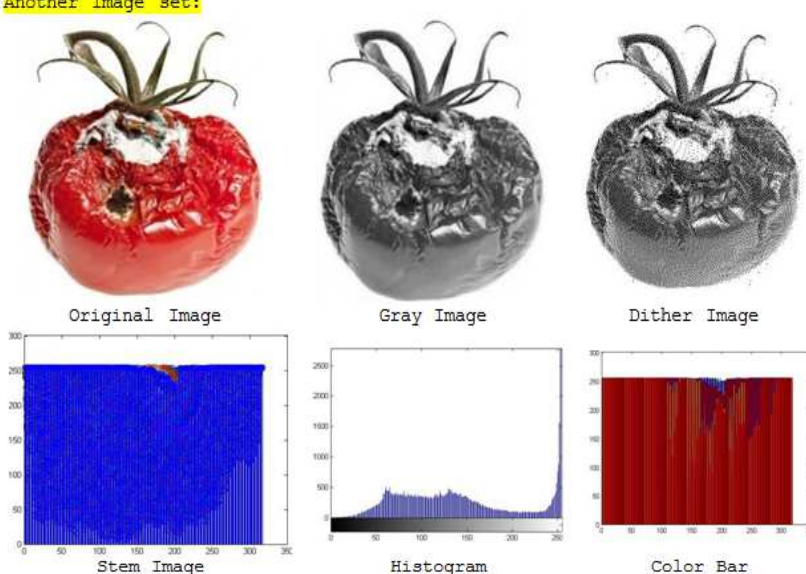

Dither Image

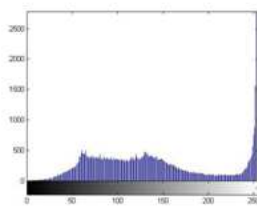

Histogram

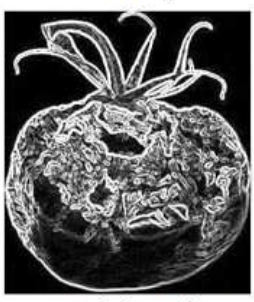

Sobel gradient

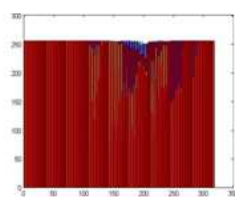

Color Bar

\section{CONCLUSIONS}

Machine vision technology can be applied to accumulate information on all these restrictions using appropriate optics and imaging system. In this paper is to new approach for detecting damaged Tomatoes. From histogram and stem value we remove the difference between the intensity among the original tomato and the spoiled tomato. 


\section{REFERENCES}

[1]. McClure JE, Morrow CT. 1987"Computer vision sorting of potatoes" ASAE Paper No.87-6501 ASAE, St. Joseph MI49085

[2]. Tao Y, Morrow CT, Heinemann PH, Sommer H J. 1995" A Fourier-based separation technique for shape grading of potatoes using machine vision" Trans. of the ASAE 38:949957

[3]. Wang X. Z., H. P. Mao, X. Han, and J. J. Yin. 2011. Vision-based judgment of tomato maturity under growth conditions. African Journal of Biotechnology, 10(18): 36163623.

[4]. Haralick, R. M., and G. S. Linda. 1992. Computer and Robot Vision, Volume I, Addison-Wesley, pp. 28-48.

[5]. Hanan, M. W., T. F. Burks, and D. M. Bulanon. 2009. A machine vision algorithm combining adaptive segmentation and shape analysis for orange fruit detection. CIGR Ejournal Vol. XI - on-line

[6]. Gerhard, J., H. M. Nielsen, and W. Paul. 2001. Measuring image analysis attributes and modeling fuzzy consumer aspects for Tomato quality grading, Research paper, research funded by the EU MACQU project contract no. AIR3-CT931603

[7]. www.mathworks.in/help/matlab.

[8]. Xu Huirong, Ye Zunzhong, Ying Yibin, "Identification of citrus fruit in a tree canopy using color information", Transactions of the CSAE, 21(5), 98-101(2005).

[9]. Otsu N., "A threshold selection method from gray-level histograms". IEEE Transactions on System Man and Cybernetics, 9(1), 62-69(1979)

[10]. Mukhopadhyay, S., Chanda, B., "Multiscale morphological segmentation of gray-scale images", IEEE Transactions on Image Processing, 12(5), 533-549(2003).

[11]. Otsu N., "A threshold selection method from gray-level histograms". IEEE Transactions on System Man and Cybernetics, 9(1), 62-69(1979)

\section{BIOGRAPHIES}

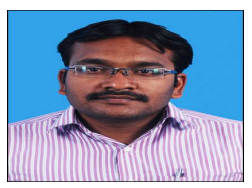

Prof. Dhanabal $\mathbf{T}$ had received his MCA Degree from SJC Institute of Technology, Chikkaballapur under VTU (visvesvaraya technological university, Belgaum) in the year 2007. He has 5 year 3 months of teaching experience. He is currently working as an Assistant Professor in Acharya Institute of Technology, Bangalore. His area of interest is Image Processing.

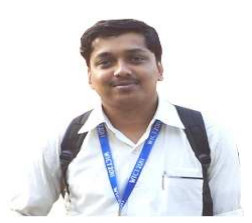

Prof. Debabrata Samanta, a member of the IAENG, Board member of the Seventh Sense Research Group Journals (SSRGJ). He obtained my MCA in the year 2010, from the Academy Of Technology, under WBUT. He has been working his $\mathrm{PhD}$ in Computer Science and Engg. from the year 2010 from
National Institute of Technology, Durgapur, India in the area of Image Processing. He is presently working as a Assistant Professor Grade III of MCA dept in Acharya Institute of Technology, Bangalore, Karnataka, India from 19th Aug,2013. His areas of interest are Artificial Intelligence, Natural Language Processing and Image Processing. He has published 47 papers in International Journals / Conferences. 